\title{
An ill-posed boundary value problem for the Helmholtz equation on Lipschitz domains
}

\author{
Wolfgang Arendt and Teresa Regińska*
}

April 16, 2008

\begin{abstract}
The paper is concerned with properties of an ill-posed problem for the Helmholtz equation when Dirichlet and Neumann conditions are given only on a part $\Gamma$ of the boundary $\partial \Omega$. We present an equivalent formulation of this problem in terms of a moment problem defined on the part of the boundary where no boundary conditions are imposed. Using a weak definition of the normal derivative, we prove the equivalence between these two problems for an arbitrary Lipschitz domain in $\mathbb{R}^{d}$. Moreover, uniqueness of the solution is proved for the general case when $\Gamma$ is a non-empty open subset of the Lipschitz boundary.
\end{abstract}

\section{Introduction}

The Helmholtz equation arises naturally in many physical applications, in particular related to acoustic or electromagnetic wave propagation. Direct problems connected with this equation are typically defined by Dirichlet or Neumann conditions on a boundary of the considered domain. The well posedness of the direct problems, i.e., properties of uniqueness, existence and stability of the solution, are well established. However, in many engineering problems the boundary conditions are underspecified or overspecified on different parts of the boundary. Such boundary value problems are in general

${ }^{*}$ The work of the second author is supported by EC FP6 MC-ToK programme TODEQ, MTKD-CT-2005-030042 
ill-posed, which means that at least one of the properties of well-posedness fails to hold (cf. [9]).

The boundary value problem considered in this paper consists in solving the Helmholtz equation $\triangle u+k^{2} u=0$ on an open domain $\Omega \subset \mathbb{R}^{d}$ with a real wave number $k$, under Dirichlet and Neumann conditions posed on $\Gamma$, a part of the boundary $\partial \Omega$ of $\Omega$. Such a problem is sometimes called a Cauchy problem for the Helmholtz equation (cf. [8], [6]) and it can be considered as the inverse problem to the Neumann (or the Dirichlet) problem for the Helmholtz equation on $\Omega$. Some reasons for an investigation of this inverse problem following from optoelectronics are explained in [10]. One possibility of solving this ill-posed problem is presented in [11]. The proposed method is based on an equivalent formulation of the boundary value problem in terms of a moment problem defined on the part $\partial \Omega \backslash \Gamma$ of the boundary. A corresponding equivalence theorem is proved there under a strong regularity assumption for the boundary. Namely, the property $\partial \Omega \in C^{1+\epsilon}$ is required. A moment problem formulation was previously applied in [4] for the boundary value problem for the Laplace equation in the two-dimensional case.

In the present paper, by using a weak normal derivative introduced in [1] (see also [3] in the present volume of Ulmer Seminare), we show the corresponding equivalence theorem for arbitrary Lipschitz domains in $\mathbb{R}^{d}$ under the assumption that $k^{2}$ is not an eigenvalue of Neumann-Laplace operator $-\Delta_{N}$ on this domain. Moreover, uniqueness of the solution of the boundary value problem is shown for the general case when $\Gamma$ is a non-empty open subset of the Lipschitz boundary $\partial \Omega$. Such uniqueness result is known for the case of a regular boundary of the class $C^{2}$ (cf. [5]).

Acknowledgements We wish to thank Ralph Chill and Mourad Choulli in Metz for helpful discussions.

\section{Weak normal derivative and Neumann-Laplace operator}

Let $V, H$ be real Hilbert spaces such that $V$ is continuously embedded in $H$. Assume furthermore that $V$ is dense in $H$. Let $a: V \times V \rightarrow \mathbb{R}$ be a continuous, symmetric bilinear form which is $H$-elliptic; i.e.

$$
a(u, u)+\omega\|u\|_{H}^{2} \geq \alpha\|u\|_{V}^{2} \quad(u \in V)
$$


where $\omega \in \mathbb{R}, \alpha>0$. The associated operator $A$ in $H$ is defined as follows:

$$
D(A)=\left\{u \in V: \exists f \in H a(u, v)=(f, v)_{H}, \forall v \in V\right\}
$$

and

$$
A u=f .
$$

Then $A$ is selfadjoint and bounded below by $-\omega$. We consider $H$ as a subspace of $V^{\prime}$ identifying $f \in H$ with the linear form $j_{f}$ given by

$$
<j_{f}, v>=(f, v)_{H} \text { for all } v \in V .
$$

Then we may define the continuous linear mapping $\Lambda: V \rightarrow V^{\prime}$ given by

$$
<\Lambda u, v>=a(u, v) \quad(u, v \in V) .
$$

Considering $\Lambda$ as an unbounded operator on $V^{\prime}$, the spectrum $\sigma(\Lambda)$ is defined as the complement of the resolvent set

$$
\varrho(\Lambda):=\left\{\lambda \in \mathbb{R}:(\lambda-\Lambda): V \rightarrow V^{\prime} \text { is bijective }\right\} .
$$

From [2], Proposition 3.10.3, we note the following

Corollary 2.1 If $A$ and $\Lambda$ are the operators defined above, then

$$
\sigma(A)=\sigma(\Lambda)
$$

Let $\Omega$ be a bounded open subset of $\mathbb{R}^{d}$, with Lipschitz boundary $\partial \Omega$. Then there exists a linear continuous mapping $\operatorname{Tr}: H^{1}(\Omega) \rightarrow L^{2}(\partial \Omega)$ such that $\operatorname{Tr}(u)=\left.u\right|_{\partial \Omega}$ for all $u \in C(\bar{\Omega}) \cap H^{1}(\Omega)$. To simplify the notation we frequently write $u$ instead of $\left.u\right|_{\partial \Omega}$. In [1] the following definition of the weak normal derivative was introduced. It requires the following usual weak definition of the Laplacian. If $w \in L^{2}(\Omega)$, then we say that $\triangle w \in L^{2}(\Omega)$ if there exists $f \in L^{2}(\Omega)$ such that

$$
\int_{\Omega} \Delta v w d x=\int_{\Omega} v f d x
$$

for all test functions $v \in \mathcal{D}(\Omega)$. Then $f$ is unique and we define $f=: \triangle w$. If in addition $w \in H^{1}(\Omega)$, then $\triangle w=f$ if and only if

$$
-\int_{\Omega} \nabla v \nabla w d x=\int_{\Omega} v f d x
$$

for all $w \in \mathcal{D}(\Omega)$. 
Definition 2.2 Let $u \in\left\{w \in H^{1}(\Omega): \triangle w \in L^{2}(\Omega)\right\}$. We say that $u$ has a weak normal derivative if there exists a function $b \in L^{2}(\partial \Omega)$ such that

$$
\int_{\Omega} \nabla u \nabla w d x+\int_{\Omega} \triangle u w d x=\int_{\partial \Omega} b w d \sigma \quad \forall w \in H^{1}(\Omega) .
$$

Then $b$ is unique and called the weak normal derivative $\frac{\partial u}{\partial \nu}=b$.

Now, let $V=H^{1}(\Omega), H=L^{2}(\Omega)$ and let

$$
a(u, v):=\int_{\Omega} \nabla u \nabla v d x, \forall u, v \in H^{1}(\Omega)
$$

The associated operator $A: D(A) \subset H^{1}(\Omega) \rightarrow L^{2}(\Omega)$ has the domain

$$
D(A):=\left\{u \in H^{1}(\Omega): \exists f \in L^{2}(\Omega) \int_{\Omega} \nabla u \nabla v d x=\int_{\Omega} f v d x \forall v \in H^{1}(\Omega)\right\} .
$$

The operator $\Lambda: H^{1}(\Omega) \rightarrow H^{1}(\Omega)^{\prime}$ is defined by (2).

Proposition 2.3 The operator $A$ is the Neumann-Laplace operator $A=$ $-\triangle_{N}$ with domain

$$
D\left(\triangle_{N}\right)=\left\{u \in H^{1}(\Omega): \triangle u \in L^{2}(\Omega), \frac{\partial u}{\partial \nu}=0 \text { on } \partial \Omega\right\},
$$

where $\frac{\partial u}{\partial \nu}$ denotes the weak normal derivative of u according to Definition 2.2. Proof: $\quad$ Let $u \in D(A)$ and $A u=f$. Then

$$
\int_{\Omega} \nabla u \nabla v d x=\int_{\Omega} f v d x \forall v \in H^{1}(\Omega)
$$

In particular, this holds for all $v$ in the test space $\mathcal{D}$. Hence $-\triangle u=f$. Introduce this into the previous equation:

$$
\int_{\Omega} \nabla u \nabla v d x+\int_{\Omega} \triangle u v d x=0 \forall v \in H^{1}(\Omega) .
$$

It means that $\frac{\partial u}{\partial \nu}=0$, by the definition of a weak normal derivative (3). Thus $u \in D\left(\triangle_{N}\right)$ and $A u=-\triangle_{N} u$.

Conversely, let $u \in D\left(\triangle_{N}\right)$ and $-\triangle_{N} u=f$. From the definition of a weak normal derivative $(3), \forall v \in H^{1}(\Omega)$

$$
\int_{\Omega} \nabla u \nabla v d x+\int_{\Omega} \triangle u v d x=\int_{\partial \Omega} \frac{\partial u}{\partial \nu} v d \sigma=0
$$


That means

$$
\int_{\Omega} \nabla u \nabla v d x=\int_{\Omega} f v d x \forall v \in H^{1}(\Omega) .
$$

So, $a(u, v)=(f, v) \forall v \in H^{1}(\Omega)$, i.e. $u \in D(A)$ and $A u=f$.

Now, given $h \in L^{2}(\partial \Omega)$, let us consider a Neumann problem for the Helmholtz equation: find $u$ in the domain of the normal derivative

$$
D\left(\frac{\partial}{\partial \nu}\right):=\left\{u \in H^{1}(\Omega): \triangle u \in L^{2}(\Omega), \frac{\partial u}{\partial \nu} \in L^{2}(\partial \Omega)\right\}
$$

such that

$$
\begin{cases}\Delta u+\mu u=0, & \text { on } \Omega \\ \frac{\partial u}{\partial \nu}=h & \text { on } \partial \Omega,\end{cases}
$$

where the normal derivative is understood in a weak sense according to Definition 2.2 , and where $\mu$ is a fixed real parameter.

Theorem 2.4 If $\Omega \subset \mathbb{R}^{d}$ is a bounded open subset with Lipschitz boundary and $\mu$ is not an eigenvalue of the negative Neumann-Laplace operator $-\triangle_{N}$, then for any $h \in L^{2}(\partial \Omega)$ the Neumann problem (7) has a unique solution in $H^{1}(\Omega)$.

Proof: $\quad$ According to Corollary 2.1, if $\mu \notin \sigma\left(-\triangle_{N}\right)$ then $\mu \notin \sigma(\Lambda)$. This means that for any linear continuous functional $F$ on $H^{1}(\Omega)$ there exists a unique solution $u \in H^{1}(\Omega)$ to the equation

$$
\int_{\Omega} \nabla u \nabla v d x-\mu(u, v)_{L^{2}(\Omega)}=F(v) \text { for all } v \in H^{1}(\Omega) .
$$

This holds also for

$$
F(v):=\int_{\partial \Omega} h v d \sigma,
$$

since the trace operator from $H^{1}(\Omega)$ into $L^{2}(\partial \Omega)$ is a linear continuous mapping for a Lipschitz domain. Applying (8) for $v \in \mathcal{D}(\Omega)$ one obtains $\triangle u+\mu u=0$. Then from the definition of the weak normal derivative we deduce that $u \in D\left(\frac{\partial}{\partial \nu}\right)$ and $\frac{\partial}{\partial \nu}=h$. Uniqueness of the solution of (7) follows from the uniqueness of (8). 


\section{The boundary value problem via a moment problem}

Throughout this section $\Omega$ is a bounded open set in $\mathbb{R}^{d}$ with Lipschitz boundary $\partial \Omega$. Moreover, $\Gamma \subset \partial \Omega$ is a given Borel set. On $\partial \Omega$ we consider the surface measure $d \sigma$. Given $f, g \in L^{2}(\Gamma)$ we consider the boundary value problem

$$
\mathbf{B P}(\mathbf{f}, \mathbf{g}) \quad \begin{cases}u \in D\left(\frac{\partial}{\partial \nu}\right), & \\ \Delta u+\mu u=0, & \text { on } \Omega \\ u=f & \text { on } \Gamma, \\ \frac{\partial u}{\partial \nu}=g & \text { on } \Gamma .\end{cases}
$$

Here $\frac{\partial u}{\partial \nu} \in L^{2}(\partial \Omega)$ has to be understood in the sense of Definition 2.2. Moreover, $\mu \in \mathbb{R}$ is a given parameter. In applications $\mu=k^{2}$ for some $k>0$. We will always assume that $\mu \notin \sigma\left(-\triangle_{N}\right)$. Then, given $\varphi \in L^{2}(\partial \Omega \backslash \Gamma)$, by Theorem 2.4 there exists a unique solution of the Neumann Problem

$$
\mathbf{N P}(\mathbf{g}, \varphi) \quad \begin{cases}u \in D\left(\frac{\partial}{\partial \nu}\right) & \\ \Delta u+\mu u=0, & \text { on } \Omega \\ \frac{\partial u}{\partial \nu}=\varphi & \text { on } \partial \Omega \backslash \Gamma, \\ \frac{\partial u}{\partial \nu}=g & \text { on } \Gamma .\end{cases}
$$

Thus BP(f,g) has a solution if and only if there exists $\varphi \in L^{2}(\partial \Omega \backslash \Gamma)$ such that the solution of $\operatorname{NP}(\mathrm{g}, \varphi)$ satisfies $u=f$ on $\Gamma$ (by which we mean more precisely $\operatorname{Tr}(u)=f d \sigma$-a.a on $\Gamma)$.

This in turn can be described by the following moment problem.

Theorem 3.1 Assume that $\mu \notin \sigma\left(-\triangle_{N}\right)$. Let $\varphi \in L^{2}(\partial \Omega \backslash \Gamma)$ and let $u$ be the solution of $N P(g, \varphi)$. Then $u=f$ on $\Gamma$ if and only if

$$
\operatorname{MP}(\mathbf{f}, \mathbf{g}) \quad \int_{\partial \Omega \backslash \Gamma} \varphi v d \sigma=\int_{\Gamma}\left[f \frac{\partial v}{\partial \nu}-g v\right] d \sigma \quad \text { for all } v \in V(\mu, \Gamma) .
$$

Here $V(\mu, \Gamma)$ consists of all $\mu$-harmonic functions in $D\left(\frac{\partial}{\partial \nu}\right)$ whose normal derivative vanishes on $\partial \Omega \backslash \Gamma$, i.e.,

$$
V(\mu, \Gamma):=\left\{v \in D\left(\frac{\partial}{\partial \nu}\right): \triangle v+\mu v=0 \text { in } \Omega, \text { and } \frac{\partial v}{\partial \nu}=0 \text { on } \partial \Omega \backslash \Gamma\right\}
$$

Of course, to say that $v \in D\left(\frac{\partial}{\partial \nu}\right)$ is $\mu$-harmonic means that $\Delta v+\mu v=0$ in the sense of distributions, or equivalently, since $v \in H^{1}(\Omega)$,

$$
\int_{\Omega} \nabla v \nabla w=\mu \int_{\Omega} v w \text { for all } w \in H_{0}^{1}(\Omega) .
$$


Proof: $\quad$ of Theorem 3.1.

a) First of all we note that for $\mu$-harmonic functions $v_{1}, v_{2} \in D\left(\frac{\partial}{\partial \nu}\right)$ one has

$$
\int_{\partial \Omega} \frac{\partial v_{1}}{\partial \nu} v_{2} d \sigma=\int_{\partial \Omega} v_{1} \frac{\partial v_{2}}{\partial \nu} d \sigma
$$

In fact, since $\triangle v_{j}=-\mu v_{j}$, by the definition of the normal derivative

$$
\int_{\Omega} \nabla v_{j} \nabla v d x-\mu \int_{\Omega} v_{j} v d x=\int_{\partial \Omega} \frac{\partial v_{j}}{\partial \nu} v d \sigma
$$

for all $v \in H^{1}(\Omega)$. Thus taking $j=1, v=v_{2}$ and $j=2, v=v_{1}$ gives the same expression on the left hand side. This proves (9)

b) Now, let $\varphi \in L^{2}(\partial \Omega \backslash \Gamma)$ and let $u$ be the solution of $N P(g, \varphi)$. Then, since $\frac{\partial u}{\partial \nu}=g$ on $\Gamma$ and by a), for all $v \in V(\mu, \Gamma)$ we have

$$
\begin{aligned}
\int_{\partial \Omega \backslash \Gamma} \varphi v d \sigma & =\int_{\partial \Omega \backslash \Gamma} \frac{\partial u}{\partial \nu} v d \sigma \\
& =\int_{\partial \Omega} \frac{\partial u}{\partial \nu} v d \sigma-\int_{\Gamma} g v d \sigma \\
& =\int_{\partial \Omega} u \frac{\partial v}{\partial \nu} d \sigma-\int_{\Gamma} g v d \sigma \\
& =\int_{\Gamma}\left(u \frac{\partial v}{\partial \nu}-g v\right) d \sigma .
\end{aligned}
$$

Thus, if $u_{\left.\right|_{\Gamma}}=f$ then $\varphi$ satisfies $\mathrm{MP}(\mathrm{f}, \mathrm{g})$. Conversely, if $\varphi$ satisfies $\operatorname{MP}(f, g)$, then it follows from the identity above that

$$
\int_{\Gamma} u \frac{\partial v}{\partial \nu} d \sigma=\int_{\Gamma} f \frac{\partial v}{\partial \nu} d \sigma
$$

for all $v \in V(\mu, \Gamma)$. By Theorem 2.4 there exists $v \in V(\mu, \Gamma)$ such that $\frac{\partial v}{\partial \nu}=u-f$ on $\Gamma$. Hence

$$
\int_{\Gamma}(u-f)^{2} d \sigma=\int(u-f) \frac{\partial v}{\partial \nu} d \sigma=0
$$

and thus $u=f$ on $\Gamma$.

Concerning existence we now have the following characterization.

Corollary 3.2 Assume that $\mu \notin \sigma\left(-\triangle_{N}\right)$. Let $f, g \in L^{2}(\Gamma)$. Then the problem $B(f, g)$ has a solution if and only if the moment problem $M P(f, g)$ has a solution $\varphi \in L^{2}(\partial \Omega \backslash \Gamma)$. 
Concerning uniqueness we obtain the following result.

Corollary 3.3 Let $\mu \in \mathbb{R} \backslash \sigma\left(-\triangle_{N}\right)$. The following assertions are equivalent:

(i) The space $\left\{v_{\mid \partial \Omega \backslash \Gamma}: v \in V(\mu, \Gamma)\right\}$ is dense in $L^{2}(\partial \Omega \backslash \Gamma)$;

(ii) if $u \in D\left(\frac{\partial}{\partial \nu}\right)$ such that $\Delta u+\mu u=0, u_{\left.\right|_{\Gamma}}=0$ and $\left.\frac{\partial u}{\partial \nu}\right|_{\Gamma}=0$, then $u=0$;

(iii) for all $f, g \in L^{2}(\Gamma)$ the problem $B P(f, g)$ has at most one solution.

Proof: The equivalence of (ii) and (iii) is obvious by linearity.

(i) $\Rightarrow$ (ii). Let $u \in D\left(\frac{\partial}{\partial \nu}\right)$ such that $u_{\left.\right|_{\Gamma}}=0$ and $\left.\frac{\partial u}{\partial \nu}\right|_{\Gamma}=0$. Let $\varphi=\left.\frac{\partial u}{\partial \nu}\right|_{\partial \Omega \backslash \Gamma}$. Then $\varphi$ satisfies $M P(0,0)$, i.e. $\int_{\partial \Omega \backslash \Gamma} \phi v d \sigma=0$ for all $v \in V(\mu, \Gamma)$. By assumption (i) this implies that $\varphi=0$. Hence $u=0$ since $\mu \notin \sigma\left(-\triangle_{N}\right)$.

(ii) $\Rightarrow($ i $)$. Let $\varphi \in L^{2}(\partial \Omega \backslash \Gamma)$ such that $\int_{\partial \Omega \backslash \Gamma} \varphi v d \sigma=0$ for all $v \in V(\mu, \Gamma)$. Then $\varphi$ satisfies $\operatorname{MP}(\mathrm{f}, \mathrm{g})$ for $f=g=0$. Let $u$ be the solution of $N P(0, \varphi)$. Then $u_{\left.\right|_{\Gamma}}=0$ by assumption. Hence $\varphi=\left.\frac{\partial u}{\partial \nu}\right|_{\partial \Omega \backslash \Gamma}=0$. This proves the density assertion (i).

Here $\Gamma \subset \partial \Omega$ is an arbitrary Borel set. If $\Gamma$ has non-empty interior with respect to the topology relative to $\partial \Omega$, then we will see in the next section that the equivalent conditions of Corollary 3.3 are satisfied.

Finally we add some remarks concerning the regularity assumptions.

1. If $\Gamma$ is the entire boundary $\partial \Omega$, then for each $g \in L^{2}(\partial \Omega)$ there is a unique solution $u \in D\left(\frac{\partial}{\partial \mu}\right)$ which is $\mu$-harmonic such that $\frac{\partial u}{\partial \nu}=g$ on $\partial \Omega$. Thus $\mathrm{BP}(\mathrm{f}, \mathrm{g})$ has a solution if and only if $f=u_{\left.\right|_{\Gamma}}$.

2. Also if $\Gamma$ is different from the entire boundary, some regularity on $f$ is needed in order a solution to exist. In fact, $f$ is the trace of an $H^{1}$-function on $\Gamma$.

3. Theorem 3.1 depends crucially on the fact that we require the solution $u$ of $\mathrm{BP}(\mathrm{f}, \mathrm{g})$ to lie in $D\left(\frac{\partial}{\partial \nu}\right)$, i.e., the normal derivative of $u$ has to exist on the entire boundary. One might also investigate a weaker assumption, namely that $\frac{\partial u}{\partial \nu}$ exists merely on $\Gamma$ in some appropriate sense. 


\section{Uniqueness of the inverse problem}

Let $\Omega \subset \mathbb{R}^{d}$ be an open, connected, bounded set with Lipschitz boundary $\partial \Omega$. Let $\Gamma \subset \partial \Omega$. Assume that there exist $z \in \partial \Omega, r>0$ such that

$$
B(z, r) \cap \partial \Omega \subset \Gamma
$$

where $B(z, r)=\left\{x \in \mathbb{R}^{d},|x-z|<r\right\}$.

Theorem 4.1 Let $\mu \in \mathbb{R}, u \in H^{1}(\Omega)$ such that $-\Delta u=\mu u$ in $\mathcal{D}(\Omega)^{\prime}$. If $\operatorname{Tr}(u)=0$ on $\Gamma$ and $\frac{\partial u}{\partial \nu} \in L^{2}(\partial \Omega)$ such that $\frac{\partial u}{\partial \nu}=0$ on $\Gamma$, then $u=0$.

Proof: Let

$$
\begin{gathered}
\widetilde{\Omega}=\Omega \cup B(z, r), \\
\widetilde{u}= \begin{cases}u & \text { on } \Omega \\
0 & \text { on } B(z, r) \backslash \Omega .\end{cases}
\end{gathered}
$$

We show that

$$
-\Delta \widetilde{u}=\mu \widetilde{u}
$$

in $\mathcal{D}^{\prime}(\widetilde{\Omega})$. This implies that $\widetilde{u}$ is analytic in $\widetilde{\Omega}$. Since $\widetilde{\Omega}$ is connected, it follows that $\widetilde{u} \equiv 0$ (because $\widetilde{u} \equiv 0$ on an open set), cf. [7], Corollary 4.4.4.

Let $v \in \mathcal{D}(\widetilde{\Omega})$. We have to show that

$$
-\int_{\widetilde{\Omega}} \widetilde{u} \triangle v d x=\mu \int_{\widetilde{\Omega}} \widetilde{u} v d x .
$$

Since $\left.\frac{\partial u}{\partial \nu}\right|_{\Gamma}=0$ and $\left.v\right|_{\partial \Omega \backslash \Gamma}=0$, we have

$$
\begin{aligned}
\mu \int_{\widetilde{\Omega}} \widetilde{u} v d x & =\int_{\Omega} \mu u v d x \\
& =-\int_{\Omega} \Delta u v d x \\
& =\int_{\Omega} \nabla u \nabla v d x-\int_{\partial \Omega} \frac{\partial u}{\partial \nu} v d \sigma \\
& =\int_{\Omega} \nabla u \nabla v d x \\
& =-\int_{\Omega} u \Delta v d x+\int_{\partial \Omega} u \frac{\partial v}{\partial \nu} d \sigma \\
& =-\int_{\widetilde{\Omega}} \widetilde{u} \Delta v d x,
\end{aligned}
$$

since $u=0$ on $\Gamma$ and $\frac{\partial v}{\partial \nu}=0$ on $\partial \Omega \backslash \Gamma$. This proves the claim.

For the case when $\partial \Omega$ is of class $C^{2}$, the uniqueness result is shown in [5], Corollary 4.4. 


\section{References}

[1] W. Arendt, G. Metafune, D Pallara and S Romanelli, The Laplacian with Wentzell-Robin boundary conditions on spaces of continuous functions Semigroup Forum, 67 (2003) 247-261.

[2] W. Arendt, Ch. J.K. Batty, M. Hieber, F. Neubrander, Vector-valued Laplace Transforms and Cauchy Problems, Birkhauser Verlag, 2001.

[3] W. Arendt, R. Mazzeo, Spectral properties of the Dirichlet-to-Neumann operator on Lipschitz domain, Ulmer Seminare, 12 (2007).

[4] J. Cheng, Y.C. Hon, T. Wei, M. Yamamoto, Numerical computation of a Cauchy problem for Laplace's equation ZAMM Z. Angew. Math. Mech., 81 (10) (2001) 665-674

[5] Mourad Choulli, Equations elliptiques et paraboliques: quelques outils et applications, Lecture Notes, Université de Metz, 2005.

[6] L. Eldén, F. Berntsson A stability estimate for a Cauchy problem for an elliptic partial differential equation Inverse Problems, 21 (2005) 16431653.

[7] L. Hörmander, The Analysis of Linear Partial Differential Operators I, Springer, Berlin, 1990

[8] V. Isakov, Inverse problems for Partial Differential Equations Springer, New York, 1998.

[9] Andreas Kirsch, An Introduction to the Mathematical Theory of Inverse Problems Springer, 1996.

[10] T. Regińska, K. Regiński, Approximate solution of a Cauchy problem for the Helmholtz equation Inverse Problems, 22 (2006) 975-989.

[11] T. Regińska, A. Wakulicz, Wavelet moment method for Cauchy problem for the Helmholtz equation, Journal of Comp. and Appl. Math, (2008), doi:10.1016/j.cam.2008.01.005,

Wolfgang Arendt, Institute of Applied Analysis, University of Ulm, D - 89069 Ulm, Germany E-mail address: wolfgang.arendt@uni-ulm.de

Teresa Regińska, Institute of Mathematics, Polish Academy of Sciences, 00-956

Warsaw, Poland E-mail address: reginska@impan.gov.pl 